\title{
Investigating the effects of 5G communication networks on smarting the urban management and sustainable economic development
}

Investigación de los efectos de las redes de comunicación 5G sobre la gestión urbana inteligente y el desarrollo económico sostenible

Author:

Mohammad Reza Zobeyri ${ }^{1, *}$

Ronak Zobeyri ${ }^{2}$

\section{SCIENTIFIC RESEARCH}

How to cite this paper:

Reaza-Zobeyri, M., Zobeyri, R. Investigating the effects of $5 \mathrm{G}$ communication networks on smarting the urban management and sustainable economic development, Tehran, Innovaciencia. 2019; 7 (2): 1-11.

DOI: http://dx.doi.org/10.15649/2346075X.758

Reception date:

Received: 11 January 2019

Accepted: 2 May 2019

Published: 25 October 2019

Keywords:

5G communications, Smart city, Smart city technologies, Urban-traffic management, Wireless Sensor Network (WSN).

\section{ABSTRACT}

This article, with a global view, explains the necessity of using smart urban technologies in the development of the infrastructure of ICT networks and in order to resolve the urban management challenges. Also, by reviewing some smart model in current modern cities, along with new generations of high-speed communications, some of the improvements made in this field are being examined. The economic view in this research has a global approach to saving energy and increasing productivity while protecting natural resources and the environment through emerging communications technologies. At the end of this research, by challenging some traditional and costly method in urban management, it is proposed to provide technological solutions to save time and money, as well as urban beautification and urbanization. With the hope that the findings of this study, though insignificant, will be the light on urban planning and urban considerations to integrate smart city technologies. It should be noted that the potential of the policymakers in the near future will be effected on creating an appropriate environment for sustainable development of the economy in national dimensions.

1* Young Researchers and Elite Club, East Tehran Branch, Islamic Azad University, Tehran, Iran.

Email: mohammadrezazobeyri@gmail.com

2 Young Researchers and Elite Club, East Tehran Branch, Islamic Azad University, Tehran, Iran. Email: ronak.zobeiri@gmail.com 


\section{INTRODUCTION}

Today, the growth and development of new generations of communications and wireless networks have facilitated and improved the aspects of urban life in modern cities. Obviously, the challenges of urban life are becoming more complicated with time and the increasing urban population and the comprehensive development of metropolitan areas. By 2050, it seems that cities will account for more than $66 \%$ of the world's population, which will cause great challenges in terms of population growth and urban development [1] The current trend of urbanization will address issues such as sustainable urban development, effective urban management, environmental sustainability and the success of urban economics among other high-level priorities for managers and policymakers. Given that the current management system is ineffective in reversing or mitigating environmental threats. Therefore, we should expect the harmful consequences of accelerated urbanization which clearly indicates the insecure and unhealthy growth of today's cities. For example, waste management problems, lack of natural resources, air pollution, health problems, population congestion, and infrastructure deterioration can be mentioned. In order to prevent the rapid growth of urbanization before it becomes a crisis, the best solution is to approach cities with an innovative way; to that end, making smart cities as a new strategy for sustainable urban development should be considered. According to the famous quotation "necessity is the mother of Innovations", it also applies for the smart cities.

Smart cities approach emerges as one of the ways to solve complex problems caused by urbanization. Smart cities strategy for solving and managing problems should be developed in a technological way. The concept of smartness in cities and megacities does not only imply the use of information and communication technology but also includes political and managerial aspects; this important issue has been neglected in many earlier studies $\stackrel{[1-7]}{ }$. Therefore, from this article, the necessity of smartness must be accompanied by a comprehensive commitment to innovation in politics, management, society, and technology. In this paper, the topic of smartness and urban management promotion has been investigated in four main topics:

- The first topic of this study is smart technology, as a block diagram of the structure of smart cities. In other words, the start of the main discussion of this article begins with the study of the "philosophy and realization of smart cities". And it goes on to show how smart electronics and telecommunication technologies, such as building bricks, can form components of smart cities as a great necessity in the near future.

- The second topic is using smart technologies for "energy saving"; the innovative creations that have been made in this area around the world have been studied by the giants of technology and their research and development.

- The third topic of the paper is the study of the effects of the $5^{\text {th }}$ generation and emerging communication technologies on "urban traffic management". In which, the topic of the architecture of advanced communication systems that control the traffic arteries continues, and how the $5 \mathrm{G}$ networks have the ability to monitor, resolve, and respond promptly to eliminate traffic jams and unexpected events.

- The fourth topic of the article is about the critique of policies adopted in "economic urban management" of Tehran as the greatest megacity of Iran, over the past few years. The study further provides solutions to beautify and save on current city costs by utilizing smart city technology. 
SMART CITY TECHNOLOGIES AS SMART CITIES BUILDER BLOCKS

So far, there have been many discussions on ways to sustainable development and quality of life in big cities, with smart management and optimizing natural resource consumption while reducing negative impacts on the environment. In all of these solutions, we can see the positive effects of citizen participation and the use of this stratum in urban development cooperation. In the meantime, smart cities claim to provide an effective model with successful managerial capabilities coupled with incentives for creativity and innovation to improve the ever-increasing quality of life while protecting the environment. Current revolutionary technologies have transformed the fundamental dimensions of urban and human life in all urban activities. Significant development of day-to-day technologies in the past decades, such as high-performance computing systems, high-speed telecommunication networks, Embedded Sensors technology, remote surgeries, which enable the Internet of Things (Io'T), all as a catalyst to form the need for smart cities to operate. Smart electrical grids, monitoring structures, health and health applications, etc., can be seen among the most commonly used smart urban technologies. Hollands, first proposed that cities should take advantage of smart urban technology through the use of a wide range of electronic and telecommunication technologies; the use of information technology to promote life and work in a region. On the other hand, the meaning of smart city is the introduction of ICT in cities and the restoration of things in a way that brings together ICT and people for creativity, innovation, science, and problem-solving ${ }^{[3]}$.

IBM, as one of the largest companies of smart city manufacturing, in 2010 upgraded the smart cities movement and the use of smart city technology to transform city systems to optimize the use of non-renewable resources. The company highlighted some of the smart city technologies, including the use of land transport by Singapore authorities to reduce traffic congestion, also, emergency handling in the Spanish city of Madrid, and the use of smart training to improve student allocation services to reduce costs in the city of New South Wales ${ }^{[2]}$.

Although innovation and technological solutions lie at the heart of the idea of smart cities, technology alone is not all the smartness. In terms of etymology, the term "Smartness" goes beyond the concept of "Intelligence", which is more spacious with the use of ICT; and therefore, one can say why political, managerial, social and environmental dimensions are all among the essential elements of the smart city. By examining technologies such as Geographic Information System (GIS), remote surgeries, wireless sensor networks (WSN), there is a question similar to the philosophical proposition of hatching eggs and chickens; whether smart cities are the ideal result of the formation of smart communities using smart technologies or vice versa. This requires more research ${ }^{[2]}$.

5G communication technology as one of the important building blocks of smart cities can be significantly affected by developing intelligent urban management. 5G technologies are the next mobile revolution which has many advantages such as improving speed and latency of the wireless networks that promoting urban facilities, high speed internet access and expanding automation which all resulting in reducing unnecessary urban traffic. Consequently, 5G network that will be deployed on 2020, enable the smart city and its functionalities ${ }^{[4]}$.

\section{Technological Solutions for Energy Savings in Smart Cities}

The content of smart cities is going beyond the use of ICT (Information and Communication Technology) and limiting the consumption of natural resources and preventing greenhouse gas emissions. Of 
course, increasing the urban efficiency, improving the quality of life, promoting commercial products and increasing environmental sustainability, etc., are the other features of smartness in urban management. One of the smart cities of East Asia is called city university of Hong Kong, which has many themes in many modern facets. There are, researchers from various high-tech fields working on providing innovative solutions to address regional and global concerns and with the goal of sustainable economic development and promoting the quality of life and the balance between the modern environment and nature.

A research team from the Electronic Engineering Department, as well as the division of building science and technology at the city University of Hong Kong, are studying to develop a smart thermostat for central air conditioning systems in buildings. In Fig. 1, this thermostat is shown to save up to $10 \%$ of energy consumption and also improve the comfort of residents inside the building. This creative technology is commercialized and expanding.
The advancement of high-frequency antennas for wireless communications is one of the main objectives of the research in the Millimeter-Wave Laboratory in the city University of Hong Kong (City-U). Experts in the lab are designing high-performance antennas to develop $5 \mathrm{G}$ wireless communications. With the emerging $5 \mathrm{G}$ communications and the transmission of signals in the millimeter-wave spectrum, they continue to develop a variety of high speed and miniaturized antennas to use in many applications. Also, in this laboratory, systems based on wireless sensor networks have been designed and fabricated that have the ability to detect smoke to prevent fire, also, to control the temperature and humidity in the building to maintain the comfort and promote the quality of life. In addition to activating an antitheft alarm, these systems monitor all of the abovementioned via the Internet. In the research teams of the department of architecture and civil engineering in City-U, have made extensive progress in improving the safety of buildings.

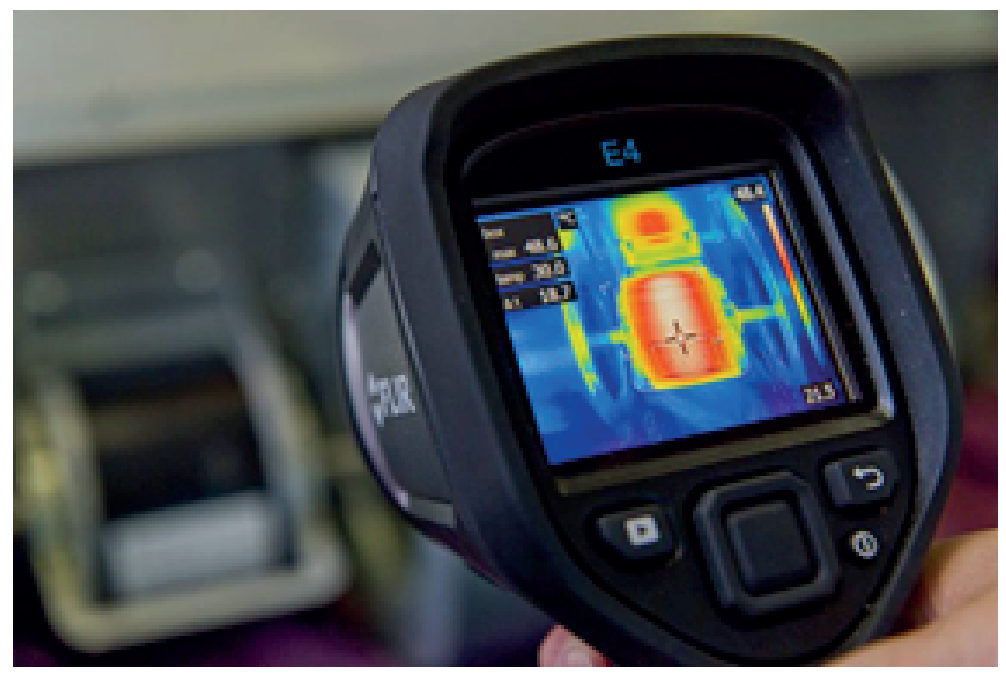

Fig. 1. Smart Thermostat for Energy Saving and Comfort Improvement in Buildings ${ }^{[5]}$

Construction monitoring systems developed in this department monitor the wind and air pressure to cope with storms and tornadoes, as well as their effects on the walls of buildings. It is noted that these knowledge-based systems are installed on a large scale commercially and in a large number of commercial buildings and privileged towers in Hong Kong and in southern China. Recent research in 
eastern Asia, especially in the south of China and Hong Kong, has attracted the support of public institutions and local companies, which previously used western tools for contractor work. In other countries, Alstom is also a global provider of smart grids, power electronics, and nanotechnology. The giant energy company supports extensive research on green energy and related projects and has set up a center for green energy research and development. In addition to Europe in the United States, General Electric has also been working towards the sustainable development of smart camps, with the aim of building up a set of smart control systems for international cooperation. These systems have the ability to reduce energy waste and more efficient use of existing energy sources ${ }^{[5]}$.

\section{Effects of 5G Technologies on Urban Traffic Management}

Nowadays, with the increase in the number of vehicles, traffic-correlated pollution, noise, visual and environmental pollution, especially in megacities, has become one of the significant challenges facing urban traffic management. Meanwhile, the use of high-speed communication technologies, smart traffic networks, public monitoring systems, emergency relief infrastructure, and more are needed. Obviously, technological issues such as vehicle locating to predict traffic, massive data processing, providing priority to emergency vehicles, and the simultaneous control of traffic lights are becoming more and more intelligence day-to-day.

With the growth of urbanization, the problem of urban traffic congestion has become a major concern in the megacities. In 2014, urban commuters in the US wasted totally 6.9 million hours, 3.1 million gallons of fuel in road traffic delays. This waste of

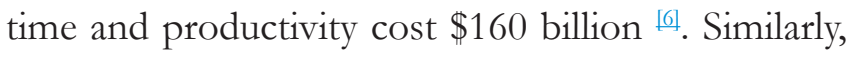
third of the world's 50 most crowded cities are in China, which traffic in these cities far worse than the United States. Experts have proposed solutions to the development of an allocated lane for Bus Rapid Transit (BRT) to expand urban transport infrastructure and reduce traffic congestion. This project has been implemented in the Tehran megacity for many years. The major disadvantage of this project is its insignificant effect on traffic jamming. Because the construction speed of BRT lanes is far less than the production speed of new vehicles [7. Therefore, the solution to the crowded urban traffic problems requires the advent of more advanced technologies. In order to effectively improving urban traffic management, at least three technological key issues must be answered:

\section{Understanding the real-time traffic situation}

Today Wireless Sensor Networks (WSNs) attracted many attentions because of the high resolution, low cost, easy installation, fast transfer rate of data which can better understand and report traffic in real-time. The duty range of smart WSN detection includes various levels of speed, direction, and location of vehicles, road throughput, weather conditions, humidity, and so on ${ }^{[6]}$. A typical urban management system based on WSNs is shown in Fig. 2. The wireless sensor networks are including a sensor node and a gateway node. Sensor nodes monitor the traffic parameters, while the gateway nodes collect the data received from sensor nodes and direct them to a base station.

As depicted in Fig. 2, information collection, data diffusion, data process and implementation of suitable action are four basic functions of WSN based urban traffic management system (WSN-UTMS). This system is equipped with Traffic Management Systems (TMCs), Road Side Units (RSUs), OnBoard Units (OBUs) in addition to WSNs to operate the above four functions as illustrated in Fig. 2. The

WSNs collect the traffic data to relay to RSU. Also, OBU is sensing the emergency vehicles. The RSU gathers data from WSNs and OBUs then, forward 
them to TMC. At last TMC receives the traffic data and analyses and ultimately makes the best intelligent decision to manage the urban road congestion and considers a priority for emergency vehicles.

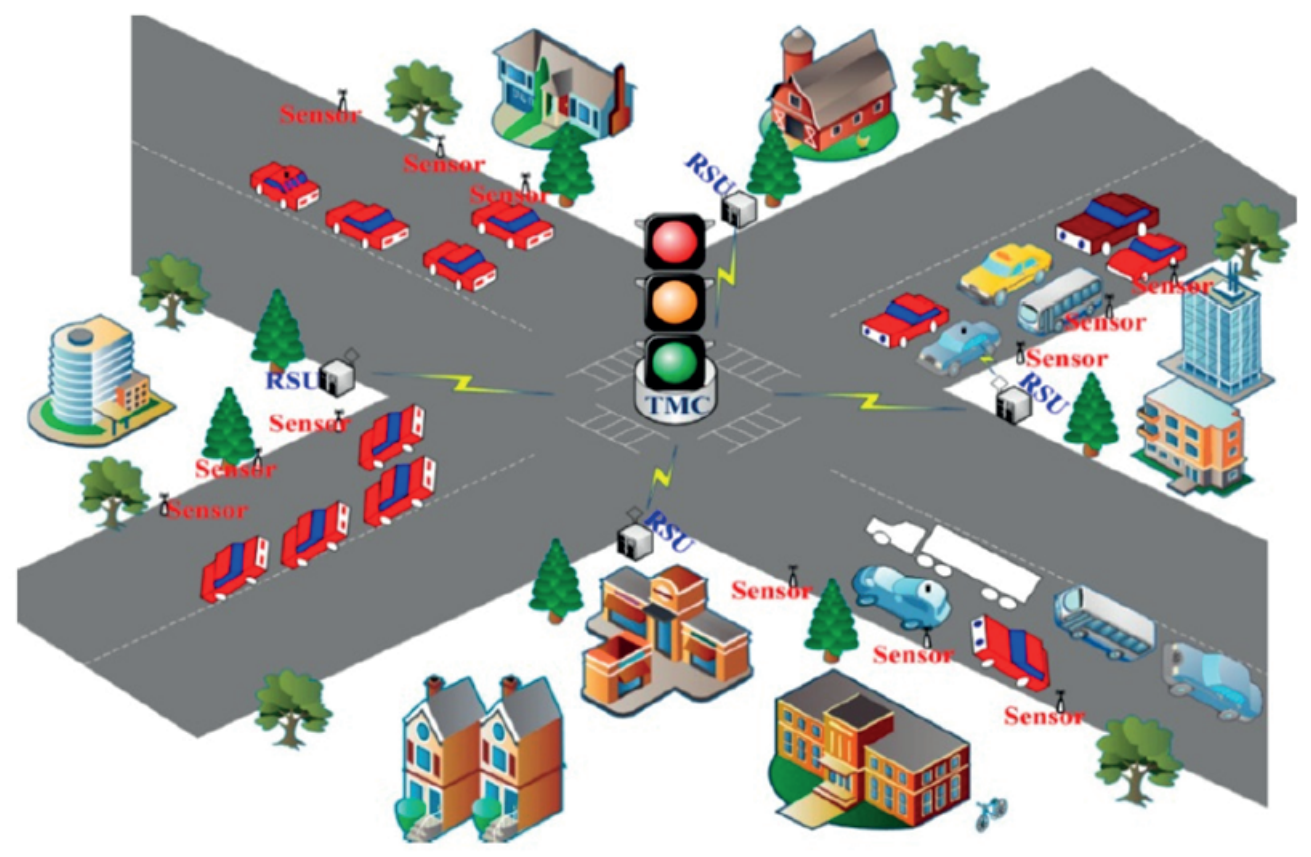

Fig. 2. WSN-based traffic management system (WSN-UTMS)

\section{Low latency response}

The sensor nodes produce a massive amount of raw data from time to time, which increases the size of the data up to the size of the petabyte over time. Due to the difference in data type, size and volume, there is no doubt that network communication bandwidth, storage capability, and data processing speed should be developed more than ever. This challenge shows the necessity of implementing and developing the next generation more than ever.

\section{Traffic prediction and real-time response}

Many traffic information helps urban planners to monitor traffic congestion, operational capability, and incident events in real-time. Traffic control systems must have the ability to respond in a realtime to respond to traffic events and make the fastest decisions based on traffic prediction algorithms to guide the traffic flow. In order to increase the productivity of existing roads, it must be considered as an essential element to design a traffic control system with intelligent machine recognition to reduce average waiting time at intersections as well as having immediate rescue accident system to provide priority for emergency vehicles. Unfortunately, current traffic control devices such as Bluetooth devices, cameras, infrared signals under the existing traditional predictor systems as well as using VANET (Vehicular Ad-Hoc Network is shown in Fig. 3) are unsuccessful to solve the above problems and avoiding road congestion. 


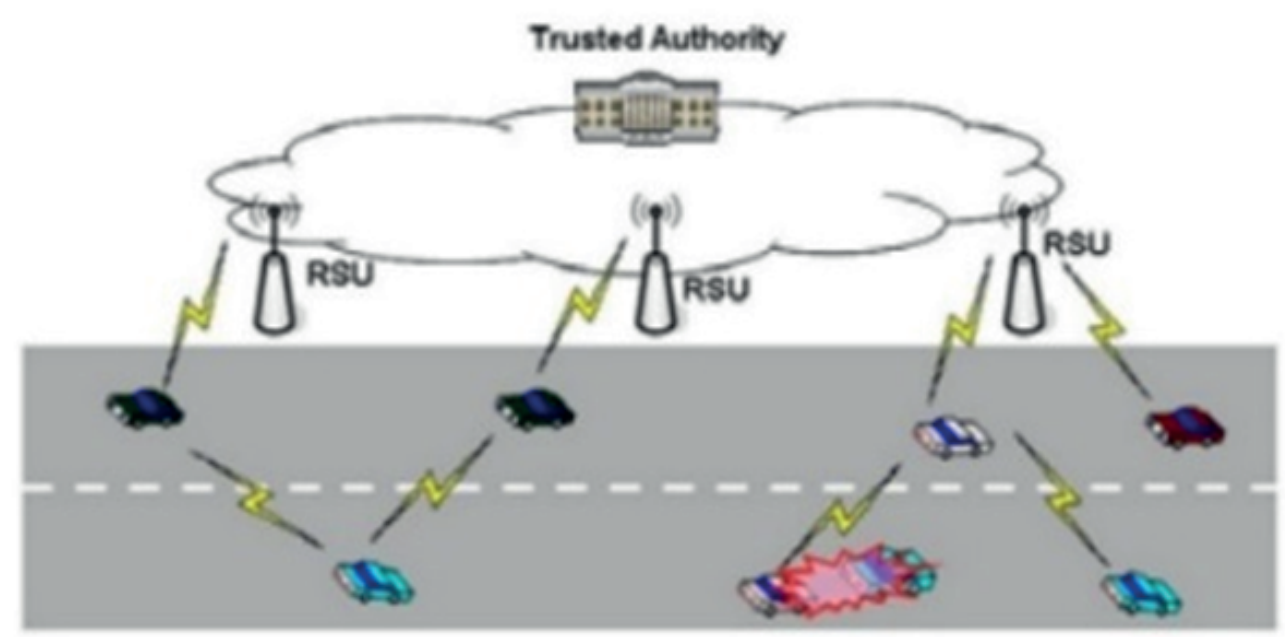

Fig. 3. Vehicle Modeling under VANETs Technology

Thanks to the rapid growth of the $5 \mathrm{G}$ communication networks, SDN (Software Defined Network) and MEC (Mobile-Edge Computing) technologies, which are expected to accelerate the growth of urban traffic management. By introducing SDN-based networks and $5 \mathrm{G}$ technologies into the new transportation network, better services with wide bandwidth and more flexibility can be obtained. The environmental sensitivity is much faster when high-resolution sensors and SDN heterogeneous networks are used. This is despite the fact that the MEC puts computing resources at the edge of the mobile vehicular network and carries out its vital mission with real-time response. In this paper, a modern architecture that combines 5G, MEC, and SDN technologies together with four-tier is investigated. The capability of this model, in addition to improving the road rescue process, is to increase its productivity in advance with features such as smart wireless sensing, high-speed communication with low latency, real-time decision-making [].

\section{Four-layer technology architecture}

Today, terrible traffic congestion makes a lot of stress for urban commuters, although emerging technologies such as $5 \mathrm{G}$ communications create a potential for reducing traffic congestion and pollutants by real-time monitoring. Based on these emerging technologies, providing a new architecture for emergency response, balancing the traffic flow and saving the time and cost of citizens is very essential. In such cases, artificial intelligence is combined with massive data mining and computational models derived from traffic data (such as machine-learning and image-processing).

Apart from the data collection, data mining and data transfer, new technologies for storing, accessing, and analyzing data are in dire need. In the four-tier architecture shown in Fig. 4, all of the necessary technologies are available simultaneously and working together easily. On the other hand, the mentioned architecture includes the environmental sensing layer, the communication layer, the MEC server layer, and the Remote Core Cloud (RCC) Server layer ${ }^{[7]}$.

\section{Environmental Sensing Layer}

In this architecture, cars play the role of smart components of the wireless sensor networks for driving the vehicle's status, which includes information about the speed, direction, also some environment information including lane, temperature, and humidity, etc. In this layer, the 
existing accuracy and integrity are due to the high sensitivity sensors reporting their status as active data at any moment. Especially the location of the vehicle, which is a very important type of traffic data that can also be estimated by the new wireless location technology.

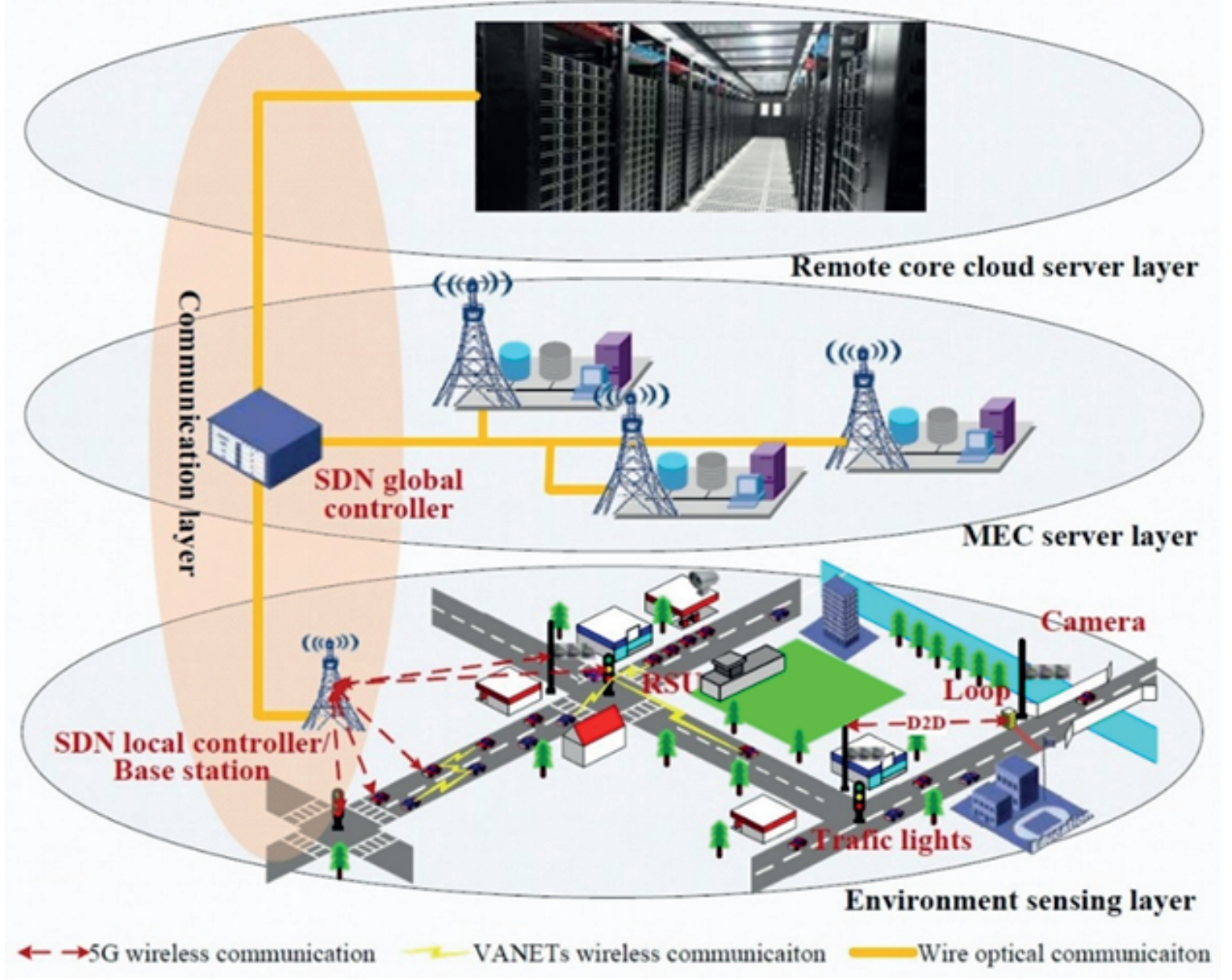

Fig. 4. Four-tier Architecture for Urban Traffic Management ${ }^{[7]}$

\section{Communication layer}

VANET is considered as one of the active technologies for connected cars but its massive traffic data has hit a lot of challenges that shown VANET unable to communicate rapidly. In order to break the traffic bottlenecks, two types of emerging networks, called $5 \mathrm{G}$ and $\mathrm{SDN}$, are considered. The $5 \mathrm{G}$ networks utilize (MIMO) multiple inputs and multiple outputs and radio cognitive technologies to achieve 1.2Gbps data rate for a moving car. SDN technology created a major revolution in networking, forwarding traffic data and making the WSNs programmable. It can be said for SDN-based networks that they are flexible, scalable, programmable and perceptible for the global network.

\section{MEC layer}

Since the beginning of using smartphones, the issue of managing the network sources was a significant challenge. By developing and complicating mobile applications, mobile network operators need to additionally deal with cloud computing issues including data processing and computing storage [8]. To perform the computation-intensive and 
latency-critical at mobile and vehicular devices a new trend in cloud computing is performed which is an increasing moving towards the network edges. By regarding that tens of millions of edge devices (such as cars and smartphones) with high-speed processors are deployed near the edge of networks. So by collecting a vast amount of idle computation devices and free storage space that distributed near the network edges, a great capacity of low-latency wireless communications and high-speed mobile computing ${ }^{[9]}$. The MEC servers are based on the $5 \mathrm{G}$ communication technology and are close to the enduser at the edges of vehicular networks and generally aimed at optimizing the mobile infrastructure service and reducing the average waiting time in the traffic flow. Due to features such as low latency, the high precision of MEC technology was proposed to improve traffic light control and to quickly accident rescue ${ }^{[8]}$.

\section{RCC server layer}

Compared to the MEC layer, this RCCS layer has more computing power and unlike the MEC server layer has wider shared resources. But the framework of the RCC server looks like MEC server and the virtual machine transfers among the RCC server and MEC server permanently. The virtual machine (VM) consists of millions of local storage with Terabytememories and a big number of high-performance virtual processors. It can be said the RCC server layer, is similar to cloud servers in Google, provides network access to a pool of shared resources including apps, servers, and storage. Therefore, the computing data will be finally centralized and stored by the RCC server layer; Traffic predictor technologies also use the Deep Learning algorithm to on-time predict the traffic flow based on the stored traffic data ${ }^{[7]}$.

\section{Investigating some Management Challenges in tehran and technological solutions}

The municipality of Tehran, as a pattern of urban management, has been experiencing accumulated problems over the past years, which has been followed by intense dissatisfaction among citizens of Tehran. According to the experts, there are two super-challenges in Tehran urban management. One of the two biggest problems is traffic congestion and the other one is air pollution. This is despite the fact that in recent years, after instability about the appointment of the Mayor of Tehran, there are some characteristics of the stability of urban management. The accumulated debt is increasing every day that is due to expansionary policies and spillovers the past years. Obviously, any institution or organization whose debt is multiplying its annual budget is considered by the economists as a bankrupt business. This is due to the fact that the Tehran Municipality in practice lacks access to powerful and stable income arteries to compensate for shortcomings and repay the existing debt. In the quickest possible action, inevitably, there should be contraction policies in urban management; in addition to preventing spills, alternative methods should be used to reduce current costs. Of course, the replacement of modern scientific methods and the use of technology in traditional ways with the aim of reducing waste of time and money is an easy and absurd task, and it is obviously not a lot of supporters in the bureaucratic bureaucracies, including our country. But today, most people welcome the use of technology for facilitating business affairs. The use of smart technologies will make the city's landscapes better and more refined. 


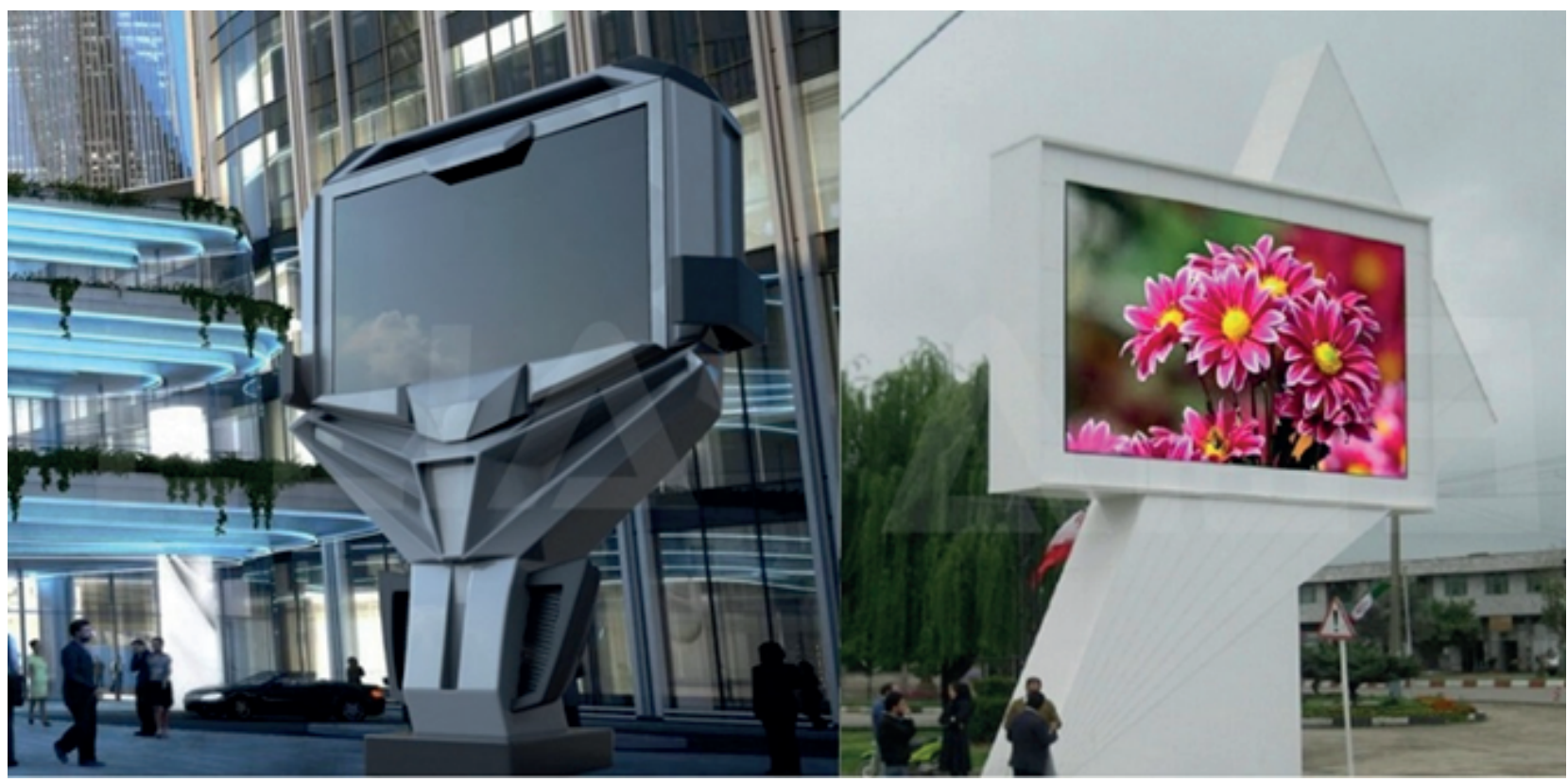

Fig. 5. Some examples of using urban smart TVs

For example, one of the things that waste cost and energy in the municipality of Tehran is printing huge amounts of banners for different ceremonies such as commemoration, congratulations, and condolences, etc. The Banners install for various occasions in the city. But they lost in a few days, and in similar cases, they will not be reusable. In some region of the municipality of Tehran, millions of US dollars spent to buy and install the banners every year. Expensive costs, constantly moving and installation on one hand, and the creation of plastic waste, on the other hand, indicate the inefficiency of this traditional method. While the more modern and economical are in common in the world that is utilizing smart TV which, in addition to its beauty is more costeffective in long-term. Fig. 5, shows two examples of using smart urban TVs instead of banners.

\section{CONCLUSION}

Any changes in society, economy, and technology are rooted in the growth of urbanization and the spread of the phenomenon of globalization. Therefore, there will be no end to the challenges of creating sustainable urban management. Solutions can be made in the light of scientific advances and the rapid growth of smart technologies such as 5G communications, but a new challenge will be created after each solution. The theme of smart cities is still under academic research, and its vision is under construction on the basis of available technologies, future discoveries, pioneer institute researches, decisions of major economic powers for global warming, approaches of business giants for investment, etc. "Smart City" is a necessity in the future, which has the ability to integrate new technologies into social systems and environmental priorities. In this paper, the phenomenon of smart cities along with the impact of significant advances in $5 \mathrm{G}$ communication technologies has been studied. And the smart technologies and systems as the main basis of smartness in the future cities are examined in four aspects. With the hope that policy-makers and managers will further develop their knowledgebased research and cutting-edge technologies into their future actions to meet the challenges of urban development. 


\section{REFERENCES}

1. Nam, T. and Pardo, T. A. (2011), "Smart City as Urban Innovation: Focusing on Management, Policy and Context," Proc. of the 5th Int. Conf. On Theory and Practice of Electronic Governance, Tallinn, Estonia, pp 26-28.

https://doi.org/10.1145/2072069.2072100

2. Yagitcanlar, T. (2015), "Smart Cities: An Effective Urban Development and Management Model," Australian Planar, 52 (55), pp 27-34. https://doi.org/10.1080/07293682.2015.1019752

3. Hollands, R. (2008), "Will the Real Smart City Stand up: Creative, Progressive, or Just Entrepreneurial?," City 12 (1), pp 302-320. https://doi.org/10.1080/13604810802479126

4. Beswick, E. and Fischer, L. (2019), "What Are the health risks associated with a $5 \mathrm{G}$ network?," https://www.euronews.com/2019/03/26/whatare-the-health-risks-associated-with-a- 5 g-network

5. City University of Hong Kong (2019), "Smart City Solutions," https://www.timeshighereducation.com/hub/city-university-hong-kong/p/ smart-city-solutions
6. Nellore, K. and Hanke, G. P. (2016), "A Survey on Urban Traffic Management System Using Wireless Sensor Networks," Sensors, 16, 157. https://doi.org/10.3390/s16020157

7. Liu, J. Wan, J. Jia, D. Zeng, B. Li, D. Hsu, C. H. and Chen, H. (2017), "High Efficiency Urban Traffic Management in Context-Aware Computing and 5G Communication," IEEE Communications Magazine, 55 (1), pp 34-40. https://doi.org/10.1109/MCOM.2017.1600371CM

8. Taleb, T. Samdanis, K. Mada, B. Flinck, H. Dutta, S. and Sabella, D. (2017), "On Multi-Access Edge Computing: A Survey of the Emerging 5G Network Edge Cloud Architecture \& Orchestration," IEEE Communications Surveys \& Tutorials, 19 (3), pp 1657-1681.

https://doi.org/10.1109/COMST.2017.2705720

9. Mao, Y. You, C. Zhang, J. Huang, K. and Letaief, K.B. (2017), "A Survey on Mobile Edge Computing: The Communication Perspective," IEEE Communications Surveys \& Tutorials, 19 (4), pp 2322-2358.

https://doi.org/10.1109/COMST.2017.2745201 